\title{
Opinión de la población graduada, con respecto a sus necesidades de formación y actualización profesional, de la carrera de Bibliotecología y Documentación, de la Universidad Nacional de Costa Rica
}

\section{Opinion of the Graduated Population about their Training and Updating Needs of the Library and Documentation Career of the National University of Costa Rica}

\author{
Máster Lucrecia Barboza Jiménez \\ Universidad Nacional, Costa Rica \\ Máster Giannina Ocampo Bermúdez \\ Universidad Nacional, Costa Rica
}

\begin{abstract}
Recibido: 14 de setiembre de $2019 \quad$ Aceptado: 06 de marzo de 2020
\end{abstract}
Publicado: 18 de junio de 2020

\begin{abstract}
Resumen
El objetivo de este estudio es identificar las opiniones de la población graduada de la carrera de Bibliotecología y Documentación de la Universidad Nacional, con respecto a su formación, y necesidades de actualización y capacitación para el fortalecimiento de competencias académicas y laborales. Esta investigación tuvo un enfoque cuantitativo y fue de tipo descriptivo; la recolección de los datos se hizo por medio de un cuestionario digital aplicado a 60 personas graduadas entre 2010 y 2018. Con los resultados se pudo obtener un perfil de la persona graduada y datos sobre la pertinencia y disponibilidad de medios y recursos para el aprendizaje en la carrera, ubicación laboral actual, desempeño profesional, expectativas de desarrollo, superación profesional y de actualización, y algunos comentarios y sugerencias de mejora.
\end{abstract}

\section{Palabras clave}

Población graduada, Encuestas de opinión, Escuela de Bibliotecología, Documentación e Información

\begin{abstract}
The purpose of this study is to identify and ask the opinion of the population that has obtained a degree in Library and Information Science from Costa Rica's National University, regarding their studies and updating needs and training, in order to strengthening their academic and labor skills. This research had a quantitative and descriptive approach. Data collection was made through a digital questionnaire that was applied to 60 persons graduated between 2010 and 2018. With the results, a profile of the graduated person could be obtained. The findings also showed information about the appropriateness and availability of means and resources during career learning, information about current work locations, about professional performance, about professional growth and improvement and some comments and suggestions for improvement.
\end{abstract}

\section{Keywords}

Graduate Population, Opinion Inquiry, School of Library, Information and Documentation 


\section{Introducción}

Asegurar la calidad en una estancia de educación superior requiere del establecimiento de niveles de compromiso y desafíos por parte de las universidades. Intervienen para ello factores tales como la comunidad educativa (entendiendo esta como estudiantes, académicos y administrativos), los planes de estudio, programas de capacitación, nuevas tecnologías, vinculación con graduados y muchos otros recursos que contribuyen en la gestión de la calidad.

Cuando la calidad está regida por estándares establecidos, se procura que los procesos realizados mantengan siempre uniformidad y constancia en cada una de las actividades que esto conlleva. Por lo tanto, es de esperar que esta calidad logre una estabilidad asociada a estrategias y mecanismos cuidadosamente estudiados y utilizando las buenas prácticas a lo largo de un proceso de evaluación y acreditación. Araneda y Pedraja (2017, p.1), mencionan que:

Efectivamente, el aseguramiento de la calidad es la actividad que logra y mantiene un cierto nivel satisfactorio en el quehacer institucional. Dicho umbral satisfactorio se logra por medio de diferentes mecanismos y sistemas y, por lo tanto, implica el cumplimiento y el mejoramiento de estándares; en orden a alinear la educación impartida con las necesidades de estudiantes, empleadores y entidades gubernamentales.

Por eso, toda la educación que recibe una persona sea formal o no, debe de ser de calidad; sin embargo, muchas instituciones luchan por ofrecer a sus clientes una serie de beneficios llamativos en busca de una mejor demanda de estudiantes. Por lo anterior, algunas carreras universitarias plantean la acreditación como una manera de asegurar el compromiso de mantener siempre la calidad en actividades relacionadas con la enseñanza, los programas académicos, la investigación, el personal académico y administrativo, así como el equipamiento y la infraestructura entre otros servicios a la comunidad estudiantil. De esta manera, la autoevaluación y la acreditación son parte fundamental del fortalecimiento de la calidad en la educación superior. Dicha aseveración se ve reforzada en la guía para la autoevaluación de carreras del SINAES en la cual se menciona que: 
En el marco de los esfuerzos por el mejoramiento de la calidad de la educación, la autoevaluación es siempre una forma interna de evaluación orientada a generar transformaciones en los distintos ámbitos de gestión institucional para fortalecerlos y propiciar una planificación sistemática de acciones de mejoramiento, así como un oportuno seguimiento de las mismas. (SINAES, 2011, p.14)

Por lo tanto, la autoevaluación se convierte en una herramienta identificadora de fortalezas y debilidades para la pronta toma de decisiones en busca del mejoramiento continuo, creando impactos positivos desde diferentes aristas como el sentido de pertenencia de toda la comunidad educativa y el incremento de la responsabilidad en la creación de procesos constantes de mejora.

El seguimiento de graduados es una de las muchas tareas que realizan las instituciones de educación superior para conocer niveles de impacto y satisfacción sobre los servicios académicos ofrecidos hacia una comunidad educativa durante el proceso académico y luego de este. Esto implica que, no solo basta con conocer la eficiencia profesional en la formación recibida, sino también la aceptación en un mercado laboral competitivo; aunado a esto, la relación entre el plan de estudios y las necesidades de los empleadores.

La Pontificia Universidad Javeriana considera que esta tarea es un insumo fundamental para la autoevaluación institucional y de los programas académicos con propósitos de acreditación. Por lo que se plantea como objetivos, en su estudio de seguimiento a recién graduados, lo siguiente:

[...] el obtener la caracterización socioeconómica, su situación laboral tanto al momento de su graduación como posteriormente, comparar la situación laboral y conocer el grado de satisfacción de los recién graduados con los servicios ofrecidos por la universidad cuando fueron estudiantes y una vez graduados. (2012, p.12)

Se vislumbra, entonces, que la información arrojada de estudios a personas graduadas se convierte en una herramienta fundamental en el autoconocimiento que una institución de enseñanza superior requiere para determinar si sus programas de estudio están en consecuencia con los perfiles de salida propuestos en los planes de estudio. 
La Escuela de Bibliotecología, Documentación e Información (EBDI) de la Universidad Nacional de Costa Rica tiene acreditada la carrera de Bibliotecología y Documentación desde el 2005. De acuerdo con esto, la EBDI requiere mantener datos actualizados de sus graduados, no solo obtenidos de los procesos de autoevaluación, sino también como una labor permanente de mejora continua.

Por esta razón, desde la actividad académica de aseguramiento de la calidad de la carrera de Bibliotecología y Documentación se estableció, en el 2018, la meta de contar con un instrumento de seguimiento a la población graduada, para conocer, entre otros aspectos, la situación laboral de estas personas, la calidad y pertinencia de la formación brindada, el nivel de inserción en el mercado laboral, los servicios o apoyos brindados, el desempeño en el mercado laboral, y la identificación de las necesidades de capacitación y actualización para el desarrollo de competencias que fortalezcan su ejercicio laboral.

Todo esto permite incorporar planes de mejora para crear y mantener un vínculo de la población graduada de la EBDI con las actividades que planifica y ejecuta la carrera, tales como talleres, cursos, conferencias, entre otras. Esta es la primera fase de varias investigaciones tendientes a desarrollar un plan de acción.

El propósito del presente artículo consiste en la presentación de los resultados de opinión de la población graduada de la EBDI, entre el 2010 y el 2018, sobre la formación recibida y las necesidades de actualización y capacitación para la mejora de su desempeño laboral.

\section{Objetivo}

Identificar las opiniones de la población graduada de la carrera de Bibliotecología y Documentación de la Universidad Nacional, con respecto a su formación y necesidades de actualización y capacitación, para el fortalecimiento de competencias académicas y laborales. 


\section{Metodología}

Esta investigación tuvo un enfoque cuantitativo y fue de tipo descriptivo; la recolección de los datos se hizo por medio de un cuestionario digital para lo cual se requirió de una revisión documental y se consultaron fuentes de diferentes universidades, tanto nacionales como internacionales, que impartieran la carrera de Bibliotecología con el fin de hallar similitudes que sirvieron como insumo para la generación de un instrumento propio acorde con los requerimientos de la investigación.

El instrumento estuvo compuesto por 46 preguntas (cerradas, abiertas y semiabiertas) dividido en siete partes: (a) perfil del graduado, b) estudios universitarios, c) pertinencia y disponibilidad de medios y recursos para el aprendizaje, d) ubicación actual laboral de las personas graduadas, e) desempeño profesional de la población graduada, f) expectativas de desarrollo, superación profesional y de actualización y g) comentarios y sugerencias.

Una vez aprobado el instrumento, se procedió a investigar sobre los posibles métodos de elaboración de encuestas en línea. Por esta razón, se estudiaron diferentes herramientas para encuestar como Google Forms, Lime Survey, Survey Monkey y E-encuesta.com. Después de un análisis de dichas herramientas, se optó por el programa Lime Survey, debido a que era el que se ajustaba más al diseño original del cuestionario y porque la Universidad contaba con una licencia para su uso.

Para la selección de la población a encuestar, la EBDI dispone de una base de datos que al 2018 contenía 310 registros con información de personas graduadas del 2010 al primer ciclo de 2018. Luego de excluir a la población graduada de diplomado, porque gran parte de ella también era graduada de bachillerato y de licenciatura, se logró incorporar a 120 participantes para el envío del cuestionario, y se establece como fecha límite tres semanas para darle respuesta. No obstante, por varios motivos (algunos ajenos a la Escuela) se dio un poco más de tiempo para que se pudiera completar dicho instrumento. 
En el transcurso del tiempo de espera y observando que aún no se lograba reunir ni una cuarta parte de los resultados, se optó por realizar llamadas telefónicas a cada uno de los participantes y se iban ingresando los datos en el sistema para facilitar el proceso de análisis de estos. Por otra parte, se visitaron algunas de las bibliotecas del Sistema de Información Documental de la Universidad Nacional (SIDUNA) para entregar en físico los cuestionarios a los participantes. Otra estrategia que se utilizó fue la de enviar a los estudiantes de nivel de licenciatura los instrumentos vía correo. Los resultados que se recopilaron de forma impresa (21 cuestionarios) también se ingresaron a la plataforma Lime Survey. El 6 de noviembre de 2018 se finalizó con el periodo de recolección de datos.

Finalmente, se recuperaron 60 cuestionarios completos y 38 incompletos, para un total de 98 instrumentos recolectados. Sin embargo, los resultados que se presentan a continuación se generaron sobre la base de 60 cuestionarios como el 100\%. Las estadísticas fueron generadas por el mismo sistema, no obstante, fue necesario volver a calcular porcentajes en algunos casos en que la base no eran las 60 respuestas. Aunado a esto se procedió a categorizar las respuestas surgidas de las preguntas abiertas para poder generar cuadros o gráficos según fuera el caso. Para la elaboración de estos cuadros y gráficos se utilizó el programa Excel.

\section{Resultados}

Se incluyeron los resultados obtenidos respecto al perfil de la persona graduada, estudios universitarios, pertinencia y disponibilidad de medios y recursos para el aprendizaje, ubicación laboral actual, desempeño profesional, expectativas de desarrollo, superación profesional y de actualización, y algunos comentarios y sugerencias de mejora.

\section{a. Perfil y estudios universitarios de las personas graduadas}

Algunos datos generales sobre las personas graduadas que permitieron establecer un perfil son los siguientes: el $80 \%$ de las personas encuestadas son mujeres y el $20 \%$ son hombres. Con respecto al estado civil, el 53,33\% son solteras, el 33,33\% son casadas, el 11,67\% están en 
unión libre y el 1,67\% divorciadas. El 55\% vive en Heredia, el 25\% en San José; el 13,33\% en Alajuela; el 3,33\% en Cartago; el 1,67 en Guanacaste y el 1,67 en Limón.

Se puede observar que, en su mayoría, las personas graduadas encuestadas son mujeres solteras que viven en Heredia. Asimismo, el 56,67\% eligió la carrera de Bibliotecología como primera opción al momento de iniciar sus estudios.

Del total de personas encuestadas, el $93,75 \%$ se graduaron de la carrera de bibliotecología y documentación y el 6,25\% de la carrera de bibliotecología pedagógica. Asimismo, el 4,55\% se egresaron del diplomado, el $51,52 \%$ de bachillerato ( $88 \%$ del énfasis de tecnologías de la información y la comunicación y $12 \%$ del énfasis de gestión de la información) y el 43,94\% de licenciatura.

Según el plan de estudio de la carrera de Bibliotecología y Documentación, el bachillerato tiene una duración de 4 años y medio y para obtener la licenciatura se requiere de 1 año y medio más, para un total de 6 años. De acuerdo con lo anterior, el 55\% de las personas encuestadas indicó que requirieron de más tiempo para poder graduarse, debido a diferentes factores: según el Gráfico 1, la mayoría de estos factores están relacionados con aspectos de la carrera (69,7\%) en lugar de situaciones personales y laborales (30,3\%).

Solamente el $23,33 \%$ ha realizado estudios en otras universidades, tales como la Universidad San Marcos, la Universidad Americana, la Universidad San José, la ULACIT, la Universidad de Costa Rica y la Universidad Latina. De este porcentaje, algunos han llevado una licenciatura en docencia y en ciencias de la información y otros han cursado maestrías en educación, docencia, currículo y administración educativa.

Del $20 \%$ que se encuentra cursando actualmente una licenciatura, el 50\% es en Bibliotecología y Documentación (Universidad Nacional), el 8,33\% en Topografía y Geodesia (Universidad Nacional), el 8,33\% en Bibliotecas Escolares y Centros de Recursos para el Aprendizaje (UNED), el 8,33\% en Bibliotecología y Nuevas Tecnologías de la Información y la Comunicación (UNED) y el 25\% en Docencia (Universidad de San José y Universidad San Marcos). 


\section{Gráfico 1 \\ Universidad Nacional \\ Escuela de Bibliotecología, Documentación e Información \\ Factores por los que las personas graduadas requirieron más tiempo para graduarse, 2018}

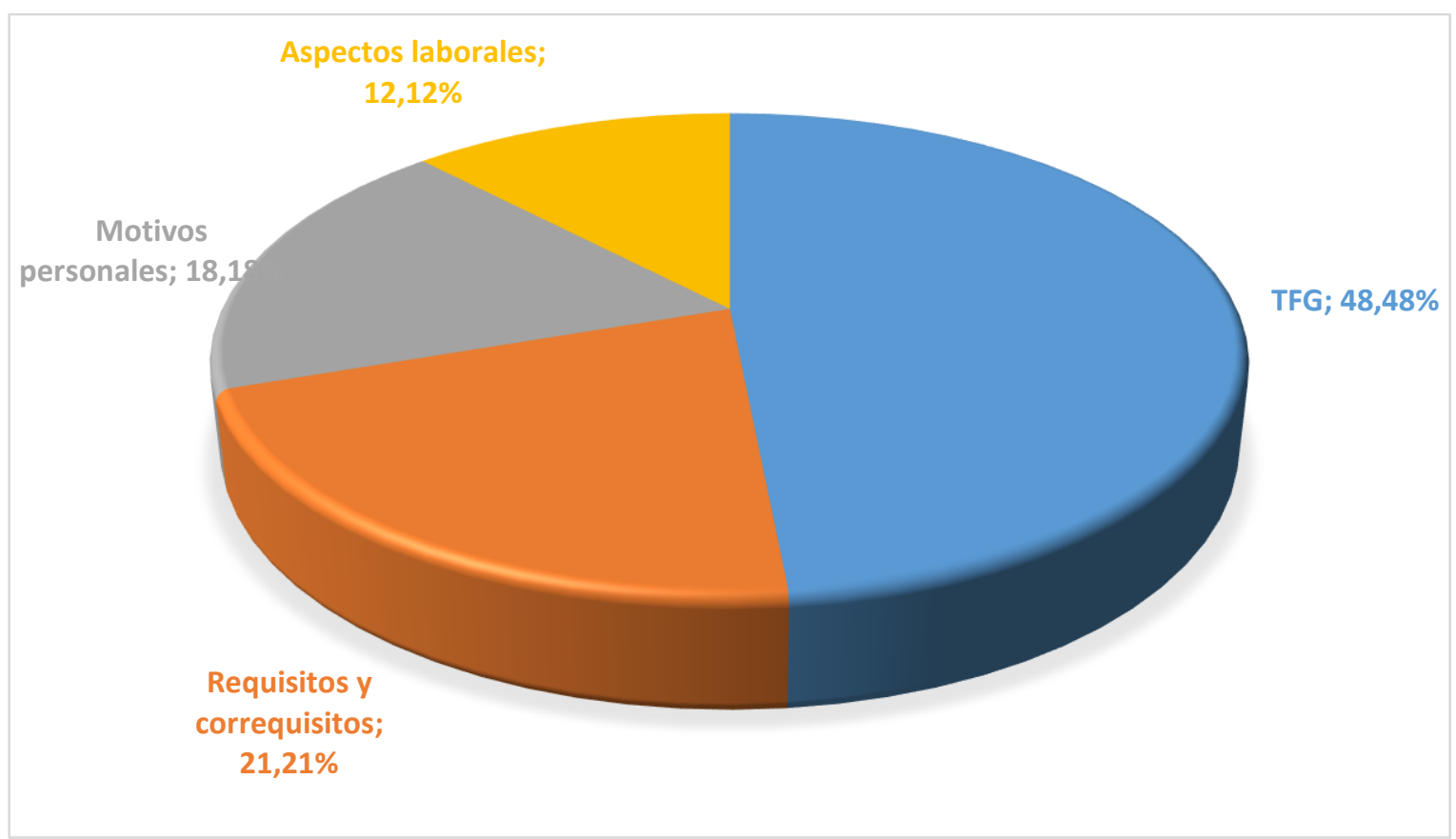

Fuente: elaboración propia de las autoras, 2019.

La actualización y la capacitación son temas de relevancia para el $61,67 \%$ de las personas encuestadas, lo cual les permite complementar la formación recibida en la Universidad Nacional. (Ver Cuadro 1).

Con respecto al manejo de herramientas para la gestión documental, la búsqueda y la recuperación de la información, el 78,33\% señala que hace uso de ellas. La herramienta más utilizada es la base de datos con un 63,83\% (ALEPH, SIABUC, KOHA, LOGICAT, OPENBIBLIO, JANIUM, EBSCO, AGRIS, American Conner), seguida por el Sistema Decimal Dewey con un $38,30 \%$, los tesauros con un $31,91 \%$ (ciencias de la salud, ciencias sociales, AGROVOC), las Listas de Encabezamiento de Materias (LEMB) con un 25,53\%, la Tabla de Notación Interna Cutter con un $12,77 \%$, las RDA y las RCAA2 con un $8,51 \%$ cada una, los catálogos públicos en línea $(6,38 \%)$, los recursos del SIDUNA $(2,13 \%)$, internet $(2,13 \%)$, 
revistas de acceso abierto (2,13\%), Google Docs (2,13\%), DSpace (2,13\%), Sistema EOS $(2,13 \%)$, IICA (2,13\%), PRIMO (2,13\%) y ELIPSIS (2,13\%).

\section{Cuadro 1}

Porcentaje de la población graduada que realiza otro tipo de actividades para complementar su formación, 2018

\begin{tabular}{|l|c|c|}
\hline \multicolumn{1}{|c|}{ Actividad } & Absoluto & Porcentaje \\
\hline Asistencia a seminarios, talleres o congresos & 27 & 72,97 \\
\hline $\begin{array}{l}\text { Asistencia a cursos de actualización o capacitación } \\
\text { en su lugar de trabajo }\end{array}$ & 24 & 64,86 \\
\hline $\begin{array}{l}\text { Asistencia a cursos de actualización o capacitación } \\
\text { en otras instituciones }\end{array}$ & 20 & 54,05 \\
\hline $\begin{array}{l}\text { Asistencia a cursos de actualización o capacitación } \\
\text { en la Universidad Nacional }\end{array}$ & 19 & 51,35 \\
\hline Se encuentra realizando investigaciones & 4 & 10,81 \\
\hline Asistencia a cursos de idiomas & 3 & 8,11 \\
\hline TOTAL & $\mathbf{3 7}$ & $\mathbf{1 0 0}$ \\
\hline
\end{tabular}

Fuente: elaboración propia de las autoras, 2019.

\section{b. Pertinencia y disponibilidad de medios y recursos para el aprendizaje}

Las personas encuestadas expresan estar satisfechas y muy satisfechas con respecto al desempeño docente $(71,67 \%)$, las condiciones de infraestructura $(71,66 \%)$ y las condiciones del equipo tecnológico (63,33\%), como se muestra en el Cuadro 2. Asimismo, consideran como buena, muy buena y excelente la calidad del plan de estudio $(79,99 \%)$, las oportunidades para participar en proyectos de investigación $(51,67 \%$ ) y el énfasis que se prestaba a la investigación dentro del proceso de enseñanza (80,01\%), según se aprecia en el Cuadro 3. 
Cuadro 2

Valoración de la satisfacción con los medios y recursos para el aprendizaje, según la población graduada, 2018

\begin{tabular}{|c|c|c|c|c|c|c|c|c|c|c|c|c|}
\hline \multirow{3}{*}{$\begin{array}{c}\text { Satisfacción } \\
\text { con los } \\
\text { medios y } \\
\text { recursos para } \\
\text { el aprendizaje }\end{array}$} & \multicolumn{12}{|c|}{ Opinión } \\
\hline & \multicolumn{2}{|c|}{$\begin{array}{c}\text { Muy } \\
\text { satisfecho }\end{array}$} & \multicolumn{2}{|c|}{$\begin{array}{c}\text { Satisfe- } \\
\text { cho }\end{array}$} & \multicolumn{2}{|c|}{$\begin{array}{l}\text { Mediana- } \\
\text { mente } \\
\text { satisfecho }\end{array}$} & \multicolumn{2}{|c|}{$\begin{array}{l}\text { Poco } \\
\text { satisfe- } \\
\text { cho }\end{array}$} & \multicolumn{2}{|c|}{$\begin{array}{c}\text { Insatis- } \\
\text { fecho }\end{array}$} & \multicolumn{2}{|c|}{ Totales } \\
\hline & $\begin{array}{l}A \\
b \\
S\end{array}$ & $\%$ & $\begin{array}{l}A \\
b \\
s\end{array}$ & $\%$ & $\begin{array}{l}A \\
b \\
S\end{array}$ & $\%$ & $\begin{array}{l}A \\
b \\
s\end{array}$ & $\%$ & $\begin{array}{l}\text { A } \\
b \\
S\end{array}$ & $\%$ & $\begin{array}{l}A \\
b \\
S\end{array}$ & $\%$ \\
\hline $\begin{array}{l}\text { Desempeño } \\
\text { docente }\end{array}$ & 18 & 30 & 25 & 41,67 & 11 & 18,33 & 4 & 6,67 & 2 & 3,33 & 60 & 100 \\
\hline Infraestructura & 20 & 33,33 & 23 & 38,33 & 13 & 21,67 & 3 & 5 & 1 & 1,67 & 60 & 100 \\
\hline $\begin{array}{l}\text { Equipo } \\
\text { tecnológico }\end{array}$ & 11 & 18,33 & 27 & 45 & 19 & 31,67 & 3 & 5 & 0 & 0 & 60 & 100 \\
\hline
\end{tabular}

Fuente: elaboración propia de las autoras, 2019.

\section{Cuadro 3}

Valoración de la calidad de los medios y recursos para el aprendizaje, según la población graduada, 2018

\begin{tabular}{|c|c|c|c|c|c|c|c|c|c|c|c|c|c|c|}
\hline \multirow{3}{*}{$\begin{array}{l}\text { Calidad de los } \\
\text { medios y } \\
\text { recursos para } \\
\text { el aprendizaje }\end{array}$} & \multicolumn{14}{|c|}{ Opinión } \\
\hline & \multicolumn{2}{|c|}{$\begin{array}{l}\text { Excelen- } \\
\text { te }\end{array}$} & \multicolumn{2}{|c|}{$\begin{array}{c}\text { Muy } \\
\text { buena }\end{array}$} & \multicolumn{2}{|c|}{ Buena } & \multicolumn{2}{|c|}{ Regular } & \multicolumn{2}{|c|}{ Mala } & \multicolumn{2}{|c|}{$\begin{array}{l}\text { Muy } \\
\text { mala }\end{array}$} & \multicolumn{2}{|c|}{ Totales } \\
\hline & $\begin{array}{l}\text { A } \\
\text { b } \\
\text { s }\end{array}$ & $\%$ & $\begin{array}{l}\text { A } \\
\text { b } \\
\text { s }\end{array}$ & $\%$ & $\begin{array}{l}\text { A } \\
\text { b } \\
\text { s }\end{array}$ & $\%$ & $\begin{array}{l}\text { A } \\
\text { b } \\
\text { s }\end{array}$ & $\%$ & $\begin{array}{l}A \\
b \\
s\end{array}$ & $\%$ & $\begin{array}{l}\text { A } \\
\text { b } \\
\text { s }\end{array}$ & $\%$ & $\begin{array}{l}\text { A } \\
\text { b } \\
\text { s }\end{array}$ & $\%$ \\
\hline $\begin{array}{l}\text { Plan de } \\
\text { estudio }\end{array}$ & 8 & 13,3 & 17 & 28,3 & 23 & 38,3 & 11 & 18,3 & 0 & 0 & 1 & 1,67 & 60 & 100 \\
\hline $\begin{array}{l}\text { Oportunidad } \\
\text { para participar } \\
\text { en proyectos } \\
\text { de investiga- } \\
\text { ción }\end{array}$ & 6 & 10 & 10 & 16,6 & 15 & 25 & 16 & 26,6 & 3 & 5 & 10 & 16,6 & 60 & 100 \\
\hline $\begin{array}{l}\text { Énfasis de la } \\
\text { investigación } \\
\text { dentro del } \\
\text { proceso de } \\
\text { enseñanza }\end{array}$ & 13 & 21,6 & 16 & 26,6 & 19 & 31,6 & 10 & 16,6 & 0 & 0 & 2 & 3,33 & 60 & 100 \\
\hline
\end{tabular}

Fuente: elaboración propia de las autoras, 2019. 


\section{c. Ubicación laboral actual de las personas graduadas}

Actualmente, la mayoría de la población graduada encuestada se encuentra trabajando. (Ver Gráfico 2). De estos graduados, el 52,83\% indica que ya lo hacían desde que estudiaban, el $26,41 \%$ señala que tiene menos de seis meses de laborar, el 15,09\% manifiesta que tiene más de un año de hacerlo y el 5,67\% entre seis meses y un año.

\section{Gráfico 2}

\section{Porcentaje de personas graduadas que trabajan o estudian, 2018}

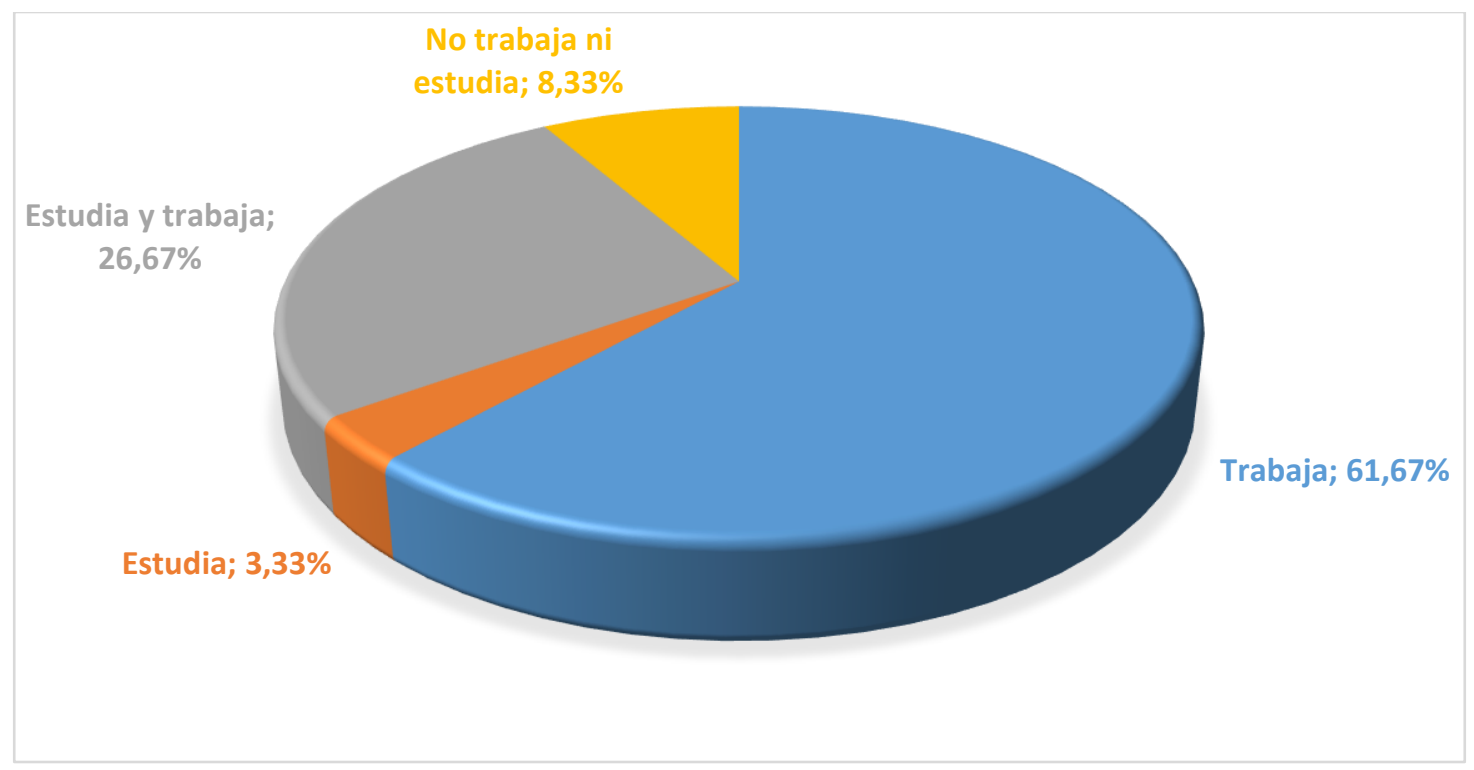

Fuente: elaboración propia de las autoras, 2019.

Sobre la relación que existe entre el trabajo que realiza y la formación recibida, el 76,67\% declara que esta relación es del 100\%; el $5 \%$ expresa que es del $80 \%$; el $1,67 \%$ que es del $60 \%$ y el $3,33 \%$ que es del $40 \%$. Solamente el $13,33 \%$ indica que su trabajo no tiene ninguna relación con su formación en bibliotecología.

Con respecto a la empresa u organismo donde trabajan, el 50\% indica que es una institución autónoma o semiautónoma, el $26,67 \%$ dice que pertenece al Gobierno Central y el 11,67\% trabaja en el sector privado. Del Cuadro 4 se desprende que los mayores empleadores de las personas graduadas encuestadas son instituciones públicas $(83,02 \%)$, especialmente la 
Universidad Nacional y el Ministerio de Educación Pública. Los lugares de trabajo se muestran en el Cuadro 4:

\section{Cuadro 4}

Lugar actual de trabajo de la población graduada, 2018

\begin{tabular}{|l|c|c|c|}
\hline \multicolumn{1}{|c|}{ Institución } & Tipo & Absoluto & Porcentaje \\
\hline Universidad Nacional & Autónoma & 24 & 45,28 \\
\hline Ministerio de Educación Pública & Gobierno Central & 15 & 28,30 \\
\hline Universidad Estatal a Distancia & Autónoma & 2 & 3,76 \\
\hline Universidad Hispanoamericana & Privada & 2 & 3,76 \\
\hline Universidad Americana & Privada & 1 & 1,89 \\
\hline BINASSS & Autónoma & 1 & 1,89 \\
\hline Universidad Católica de Costa Rica & Privada & 1 & 1,89 \\
\hline Universidad Escuela Libre de Derecho & Privada & 1 & 1,89 \\
\hline Universidad Técnica Nacional & Autónoma & 1 & 1,89 \\
\hline Universidad Iberoamérica & Privada & 1 & 1,89 \\
\hline IICA & Autónoma & 1 & 1,89 \\
\hline Centro Cultural Costarricense Norteamericano & Privada & 1 & 1,89 \\
\hline Ministerio de Cultura & Gobierno Central & 1 & 1,89 \\
\hline Colegio Universitario de Cartago & Semi autónoma & 1 & 1,89 \\
\hline \multicolumn{2}{|c|}{ TOTAL } & $\mathbf{5 3}$ & $\mathbf{1 0 0}$ \\
\hline
\end{tabular}

Fuente: elaboración propia de las autoras, 2019.

Con respecto al tiempo que cada persona tiene de trabajar para la institución respectiva, el $35,85 \%$ tiene entre uno y cinco años, el $26,41 \%$ entre 11 a 20 años, el 13,21\% menos de un año, el 13,21\% entre 6 y 10 años y el 9,43\% más de 20 años. Lo anterior demuestra que la población graduada goza de estabilidad laboral, lo cual es congruente con el hecho de que una gran mayoría trabaja para el sector público. En el Cuadro 5 se muestran los medios que las personas graduadas utilizaron para obtener el empleo actual.

En relación con las competencias que las personas graduadas debían tener para la contratación laboral, el $83,33 \%$ indicó el título profesional, el 56,67\% actitudes y habilidades sociocomunicativas, el $43,33 \%$ experiencia, el $21,67 \%$ un examen de selección, el $15 \%$ un segundo idioma, el $18,33 \%$ otras competencias y el $8,33 \%$ señaló que ninguna. 


\section{Cuadro 5}

Medios para obtener el empleo según la población graduada, 2018

\begin{tabular}{|l|c|c|}
\hline \multicolumn{1}{|c|}{ Medios } & Absoluto & Porcentaje \\
\hline Contactos personales & 20 & 33,33 \\
\hline Medios de comunicación masiva & 13 & 21,67 \\
\hline Bolsa de trabajo de la Escuela & 8 & 13,33 \\
\hline Colegio Profesional en Bibliotecología & 1 & 1,67 \\
\hline Otro & 18 & 30,00 \\
\hline TOTAL & $\mathbf{6 0}$ & $\mathbf{1 0 0}$ \\
\hline
\end{tabular}

Fuente: elaboración propia de las autoras, 2019.

Con respecto al título profesional, al $32 \%$ le solicitaron el bachillerato, al $14 \%$ el diplomado y al $10 \%$ la licenciatura; el $44 \%$ no indicó el grado. Las actitudes y habilidades socio-comunicativas valoradas fueron: dinamismo, servicio al cliente, solución de conflictos, fluidez oral, expresión oral y escrita, liderazgo, manejo de usuarios, comunicación, presentación personal y creatividad. La experiencia solicitada varía entre uno a dos años. El examen de selección se refirió a pruebas psicológicas y técnicas y el conocimiento de un segundo idioma al inglés. Las otras competencias solicitadas fueron en cuanto a sistemas de información, manejo de herramientas tecnológicas, manejo de bases de datos, ALEPH.

Según la población graduada, el inglés es otro de los idiomas que utiliza en su trabajo (56,67\%), otras personas indicaron que el francés $(6,67 \%)$, el italiano $(1,67 \%)$ y el portugués $(1,67 \%)$. El $43,33 \%$ indicó que no requiere de otro idioma, además del español, para desarrollar su trabajo. Estos idiomas se utilizan para desempeñar actividades laborales relacionadas con la lectura (96,67\%), la escritura (95\%), la escucha $(93,33 \%)$ y el habla $(91,67 \%)$.

En cuanto al salario mensual, la mayoría percibe menos de $\$ 500.000$ aunque once personas señalaron que reciben un salario entre $\$ 800.000$ y $\$ 1.000 .000$, lo cual se muestra más claramente en el Gráfico 3. Además, el 73,33\% funge como subordinado, el 20\% tiene un mando medio o jefatura, el $5 \%$ es trabajador independiente y el 1,67\% trabaja en la alta gerencia 0 dirección. El $51,67 \%$ de los encuestados tiene propiedad, el $26,67 \%$ es interino y el $8,33 \%$ 
trabaja por contrato; el restante $13,33 \%$ no se encuentra trabajando o no responde. Por último, el nivel de satisfacción con el trabajo actual se puede apreciar en el Gráfico 4.

\section{Gráfico 3}

Salario mensual según la población graduada, 2018

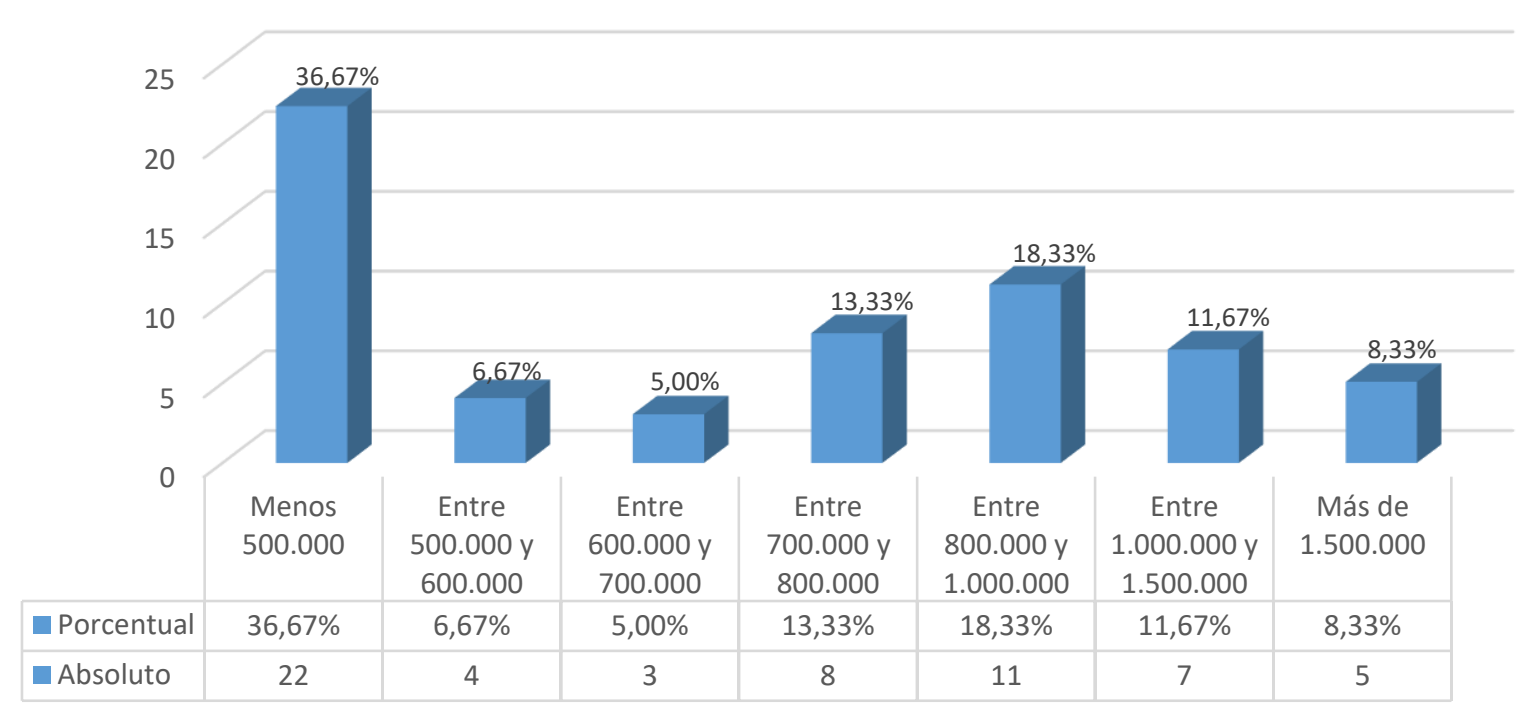

Fuente: elaboración propia de las autoras, 2019.

\section{Gráfico 4}

Nivel de satisfacción con el trabajo actual, según la población graduada, 2018

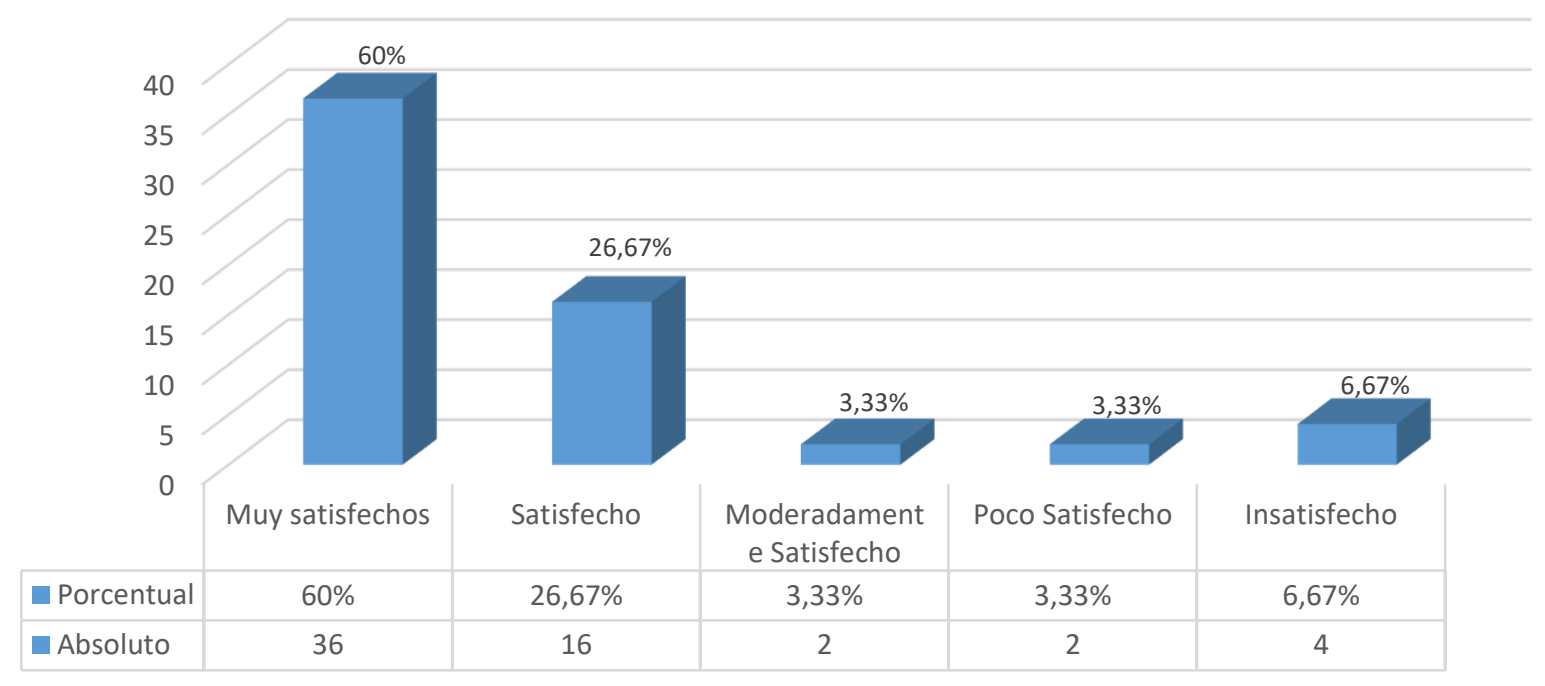

Fuente: elaboración propia de las autoras, 2019. 


\section{d. Desempeño profesional de las personas graduadas}

En este apartado se pretende determinar la coherencia entre la formación y el tipo de empleo de las personas graduadas. En este sentido, en el Gráfico 5 se puede apreciar las respuestas de las personas graduadas sobre la relación de la formación académica recibida y las actividades que realizan en el trabajo, y en el Gráfico 6 sobre la opinión en cuanto a la utilidad de las prácticas profesionales para el desarrollo profesional y laboral.

\section{Gráfico 5}

Relación entre la formación recibida y las actividades realizadas en el trabajo, según la población graduada, 2018

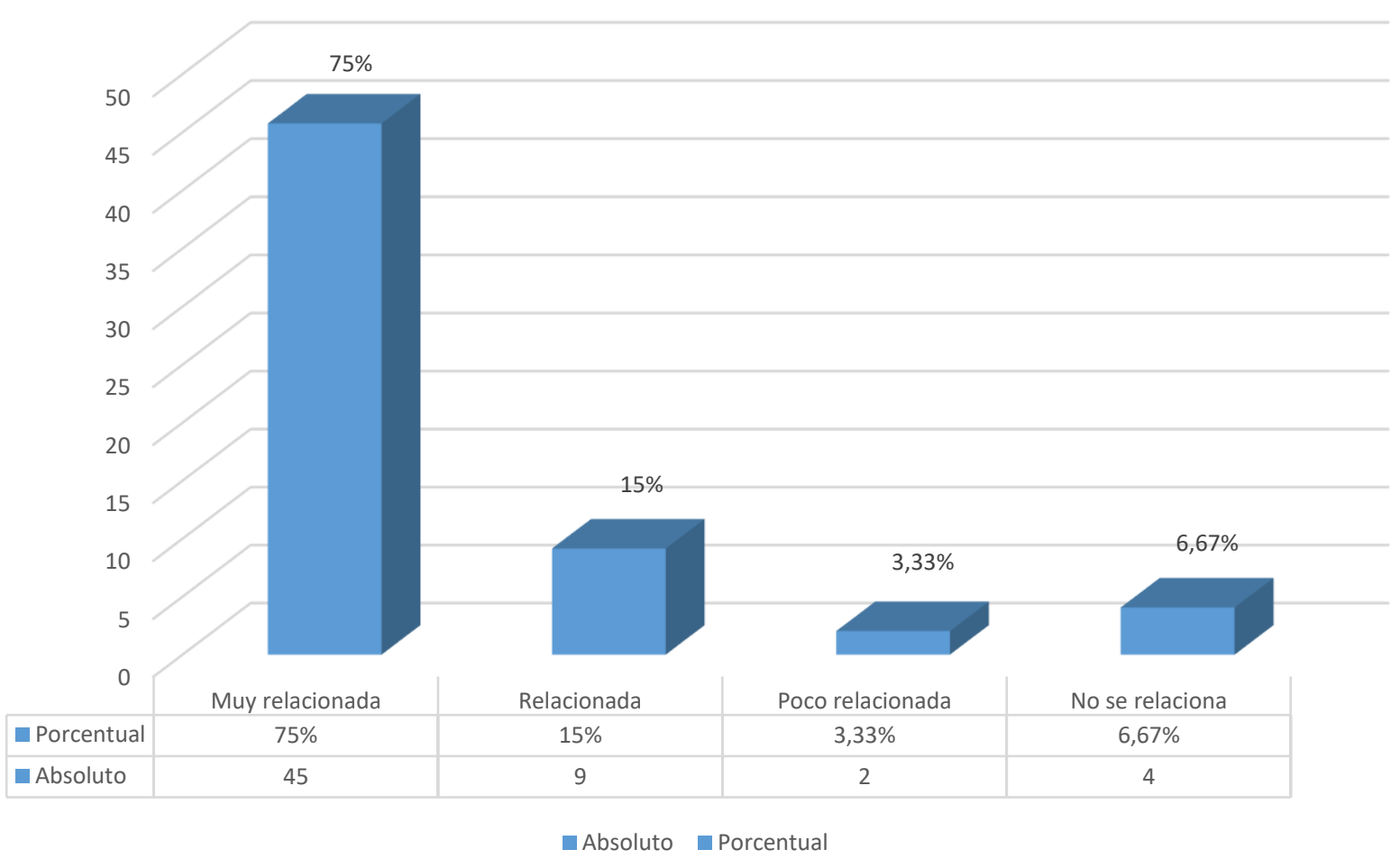

Fuente: elaboración propia de las autoras, 2019. 


\section{Gráfico 6}

\section{Opinión en cuanto a la utilidad de las prácticas profesionales para el desarrollo} profesional y laboral, según la población graduada, 2018

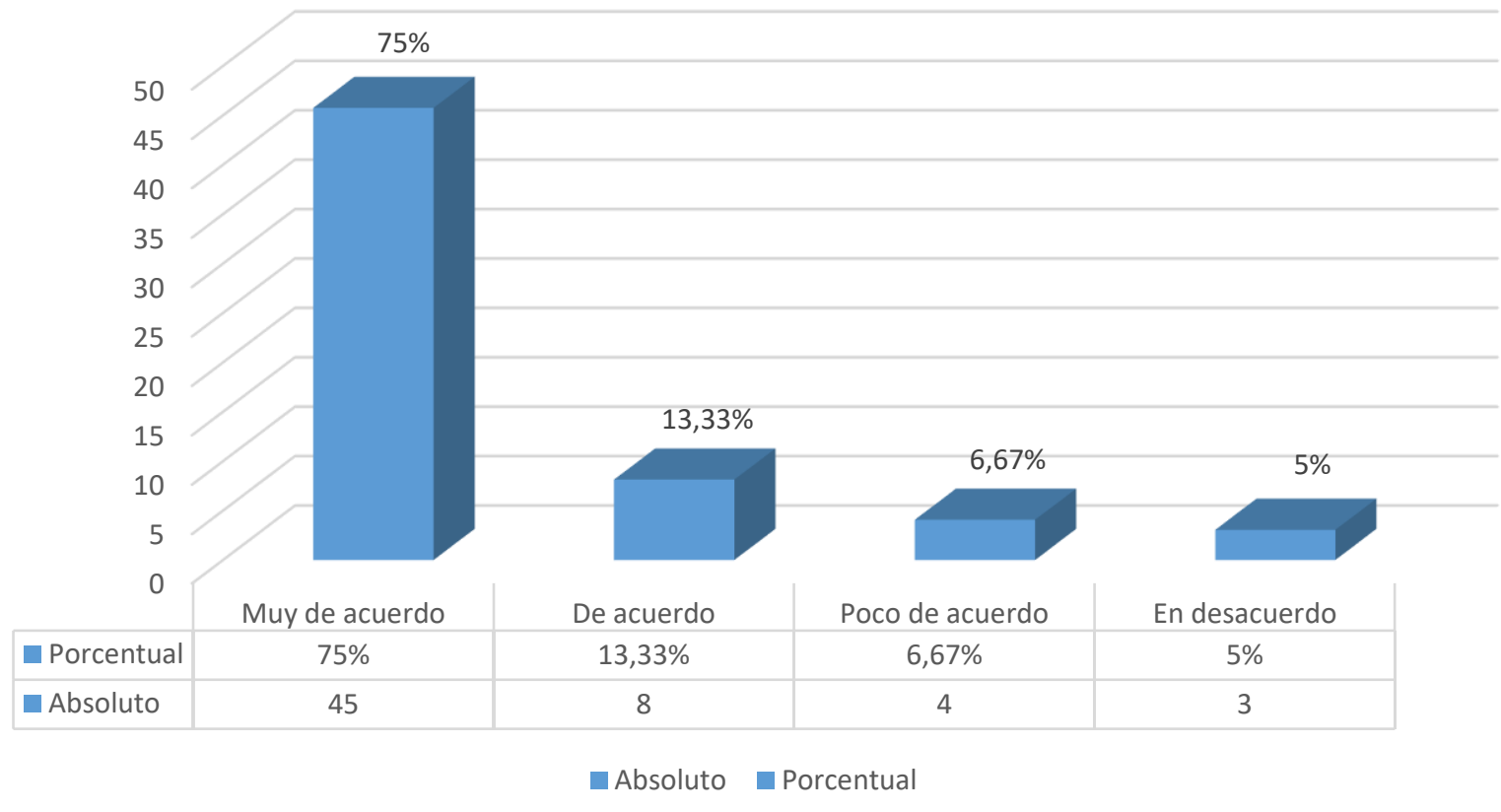

Fuente: elaboración propia de las autoras, 2019.

\section{e. Expectativas de desarrollo, superación profesional y de actualización}

El $88,33 \%$ de las personas graduadas elegirían otra vez la Universidad Nacional si tuvieran que cursar de nuevo la carrera. De los graduados que respondieron afirmativamente, adujeron diversos motivos como los que se muestran en el Cuadro 6.

\section{Cuadro 6}

Motivos para elegir la UNA para volver a cursar la carrera, según la población graduada, 2018

\begin{tabular}{|l|c|c|}
\hline \multicolumn{1}{|c|}{ Motivos } & Absoluto & Porcentaje \\
\hline Calidad & 30 & 50 \\
\hline Conveniencia & 13 & 21,67 \\
\hline Sistema & 9 & 15 \\
\hline Prestigio & 5 & 8,33 \\
\hline Por gusto & 5 & 8,33 \\
\hline
\end{tabular}

Fuente: elaboración propia de las autoras, 2019. 
Las personas graduadas que respondieron de una forma negativa $(11,67 \%)$ aducen esta respuesta al tiempo que les tomó el estudio y la distancia para llegar a la universidad. Cabe mencionar que, al ser una pregunta abierta, permitió que una persona pudiera responder varias categorías. En cuanto a la "Calidad", los encuestados expresaron que tanto los profesores como la formación recibida son una parte fundamental en el proceso de la enseñanza. Así como la calidad de las instalaciones y la incorporación de las tecnologías.

La "Conveniencia" en este estudio se refiere a algunos aspectos tales como la cercanía, las facilidades de pago y los horarios. Dentro de la categoría de "Sistema", se pueden mencionar: universidad con enfoque humanista y los valores. Asimismo, el $86,67 \%$ de las personas graduadas volverían a estudiar la carrera de Bibliotecología; en cuanto a los motivos de esta afirmación se tienen los siguientes: afinidad (48,33\%), mercado laboral (20\%), plan de estudios (10\%) y naturaleza de la disciplina (10\%). La carrera de bibliotecología ha sido una disciplina en la que la mayoría de las personas que la estudiaron han logrado ubicarse en el campo laboral, razón por la cual es uno de los mayores intereses por los cuales se escoge la carrera como primera opción. En el caso de las personas graduadas que respondieron a la categoría de afinidad se refería principalmente al gusto por la carrera. Por otra parte, al 91,67\% le interesa acceder a cursos de actualización o de alguna especialidad. El Cuadro 7 muestra la diversidad de temas que apuntó la población graduada.

\section{Cuadro 7}

Temas de interés para actualización profesional, según la población graduada, 2018

\begin{tabular}{|l|c|c|}
\hline \multicolumn{1}{|c|}{ Temas } & Absoluto & Porcentaje \\
\hline Tecnología & 29 & 48,33 \\
\hline RDA & 26 & 43,33 \\
\hline Fomento a la lectura & 8 & 13,33 \\
\hline Bases de datos & 7 & 11,67 \\
\hline Gestión de la información y el conocimiento & 7 & 11,67 \\
\hline Gestores bibliográficos & 4 & 6,67 \\
\hline Otros & 11 & 18,33 \\
\hline
\end{tabular}

Fuente: elaboración propia de las autoras, 2019. 
En la categoría de "Tecnología" la población graduada ha apuntado las siguientes subcategorías: tendencias y herramientas tecnológicas para la gestión. En materia de "Bases de datos" las respuestas de mayor frecuencia fueron: la utilización de softwares para el tratamiento de la información y al desarrollo de colecciones. Al realizar una categorización para referirse a los "Gestores bibliográficos" se pueden mencionar algunos como la búsqueda de información y estrategias de búsqueda. En la opción "Otros" se pueden ubicar temas como: bibliometría, conservación, investigación, diseño gráfico, idiomas, mercadeo, edición de revistas, ALFIN, educación y propiedad intelectual.

\section{f. Comentarios y sugerencias}

En esta sección, las personas graduadas externaron sus opiniones o recomendaciones para mejorar la formación profesional. Las respuestas se pueden categorizar en grandes áreas como las siguientes:

- Actualización: implementar idiomas, tecnologías (programas y equipo), cursos y contenidos del plan de estudio; diversificar los énfasis, diferenciar los cursos de bachillerato y licenciatura, dar seguimiento al avance de cada estudiante y a los profesores.

- Enfoque en pedagogía: ofrecer cursos de fomento a la lectura y desarrollo de habilidades lectoras, actividades de capacitación y actualización para las personas graduadas en este tema.

- Participación de la población graduada: ofertar cursos de actualización y grupos de apoyo con esta población.

- Investigación: involucrar al estudiantado en investigaciones y publicaciones, crear un fondo especial de investigación, fortalecer los cursos de investigación del plan de estudio desde el inicio de la carrera.

- Personal docente: mostrar más exigencia por parte de la Escuela para la contratación de profesores, ofrecer más apoyo al estudiantado de licenciatura, mejorar las relaciones profesor-estudiante, y ofrecer más interacción docente-estudiante.

- Estudios de posgrado: implementar un plan de estudio de posgrado o establecer convenios con otras universidades y ejecutar una maestría en tecnologías, pues la 
carrera debería reconocer qué campo necesitan las empresas para capacitar nuevos bibliotecarios.

- Oferta académica y cursos de actualización: apoyar a los estudiantes para capacitaciones en el exterior, incluir cursos de conservación y restauración, buscar la innovación, implementar cursos virtuales o bimodales, desarrollar más cursos de catalogación, regionalizar la educación para llevar la formación bibliotecológica al Caribe, conocer más bases de datos, realizar talleres sobre el desarrollo de aplicaciones en el celular acerca del tema de bibliotecología, cambiar la metodología, dar énfasis en servicio al cliente, implementar cursos de idioma y lenguaje por señas, incluir más tecnología e investigación, ofrecer cursos más flexibles y más agiles en los niveles básicos, capacitar en la elaboración de ensayos.

- Proyectos y prácticas: valorar la pertinencia de los proyectos, solicitar más apoyo a la BIMIAL, aportar más trabajo de campo donde se puedan ver las necesidades de comunidades y unidades de información, involucrar más los proyectos de extensión y dirigidos a poblaciones vulnerables.

\section{Conclusiones}

Luego del análisis de la información recolectada se concluye lo siguiente:

- La población graduada sigue siendo en su mayoría mujeres. Asimismo, la Universidad Nacional, al estar ubicada en Heredia, atrae en mayor medida a la población de esta provincia.

- Existe una tendencia de seleccionar la carrera de Bibliotecología como una alternativa o trampolín para ingresar a la Universidad con la esperanza de luego hacer cambio de carrera. Además, los porcentajes de elección de la disciplina como primera opción están en aumento en los últimos años.

- La gran mayoría se gradúa de la carrera de Bibliotecología y Documentación porque es la ofertada regularmente. De la población encuestada, se aprecia que poco más de la mitad son graduados de bachillerato, y es el énfasis de tecnologías de la información y la comunicación el más atractivo para el estudiantado. 
- Las personas que requirieron más tiempo del estipulado en el plan de estudio para graduarse, fue debido a factores relacionados con la carrera, tales como los requisitos y correquisitos establecidos en el plan de estudio y aspectos vinculados con los trabajos finales de graduación.

- Las valoraciones sobre aspectos de la Escuela, la carrera y el personal docente son muy positivas, aunque hay sugerencias de mejora por parte de las personas graduadas, tales como actualización del plan de estudio y del equipo tecnológico; revisión de los contenidos y cursos para evitar repeticiones de temas, además de ofrecer cursos de tecnología más actualizados, mejores relaciones e interacción profesor-estudiante, fortalecimiento de los cursos de investigación, entre otras.

- Una de las características más importantes de resaltar de la carrera es que dispone de una amplia demanda laboral, que le permite a la población estudiantil insertarse en el mercado de trabajo desde que se está estudiando. Esto queda de manifiesto con las respuestas de más del $80 \%$ de las personas encuestadas que afirman la existencia de una relación entre el trabajo que realizan y su formación en Bibliotecología. Además, las labores que realizan en sus respectivos trabajos también están muy relacionadas con la formación académica recibida.

- Otro aspecto digno de resaltar es el hecho de que la mayoría de las personas encuestadas trabajan para instituciones públicas (autónomas, semi autónomas y gobierno central), de las que resaltan la Universidad Nacional y el Ministerio de Educación Pública; estos resultados coinciden plenamente con los porcentajes de personas encuestadas que disfrutan de estabilidad laboral. El tipo de institución en la que trabajan los graduados debe ser tomada en cuenta a la hora de plantear algunos contenidos de los programas de curso que podrían ayudar al estudiantado en su futuro profesional, o en los cursos de capacitación y actualización que se puedan ofertar.

- Resulta primordial impulsar las acciones institucionales (bolsas de empleo, colegio de profesionales, etc.) como puente para que las personas graduadas puedan colocarse en el mercado de trabajo, ya que los resultados obtenidos demuestran que los encuestados lograron acceder a sus respectivos trabajos gracias, en mayor medida, a contactos personales y a los medios de comunicación masiva. 
- El título profesional sigue siendo el principal requisito que solicitan las organizaciones para conceder a un puesto de trabajo; no obstante, las actitudes y habilidades sociocomunicativas, la experiencia y el conocimiento de otro idioma, también son competencias muy deseables para los empleadores. Sobre las actitudes (presentación personal) y habilidades (dinamismo, servicio al cliente, solución de conflictos, fluidez oral, expresión oral y escrita, liderazgo, manejo de usuarios, comunicación y creatividad) es importante retomar estas respuestas en un futuro plan de capacitación y actualización a la población graduada.

- Las personas graduadas apuntan como principales temas de interés para cursos de actualización los siguientes: tecnologías en cuanto a tendencias y herramientas para la gestión y sobre la norma de catalogación internacional RDA. Esto es congruente con las exigencias actuales del mercado laboral, debido a que, en el caso de las RCAA2, estas resultaron insuficientes para procesar la diversidad de materiales que surgieron gracias al acelerado avance tecnológico, lo que generó la necesidad de crear un nuevo código de catalogación llamado RDA. Con respecto a la gestión, siempre aparecen nuevas herramientas para la búsqueda, procesamiento, organización y recuperación de la información que los profesionales en bibliotecología deben conocer y manejar para mantener vigentes sus respectivas unidades de información.

- Es significativo rescatar que tanto la Universidad Nacional como la carrera de Bibliotecología y Documentación son valoradas positivamente por las personas graduadas encuestadas, tanto por su calidad y prestigio en el caso de la primera, como por el mercado laboral y el plan de estudios de la segunda, entre otras razones.

Para finalizar, y a modo de conclusión general, estos resultados permiten a la EBDI tomar decisiones y ejecutar acciones importantes en beneficio de la comunidad educativa. Todas las observaciones externadas por las personas graduadas son oportunidades de mejora que se verán reflejadas en futuros proyectos vinculados a los procesos de aseguramiento de la calidad y como complemento en la autoevaluación de la carrera para la mejora continua. 


\section{Referencias bibliográficas}

Araneda, C. y Pedraja, L. (2017). Aseguramiento de la calidad y la investigación en las universidades. Ingeniare. Revista chilena de ingeniería, 25(4), 564-565. Recuperado de: https://dx.doi.org/10.4067/S0718-33052017000400564

Pontificia Universidad Javeriana. (2012). Estudio de seguimiento a recién egresados de programas académicos de pregrado de la sede central. Bogotá, D. C.: Editorial Pontificia Universidad Javeriana. Recuperado de: https://bit.ly/2kV2hAX

Sistema Nacional de Acreditación de la Educación Superior. (2011). Guía para la autoevaluación de carreras. San José, C.R.: SINAES.

\section{Datos de las autoras}

Lucrecia Barboza Jiménez: Licenciada en Bibliotecología con énfasis en Ciencias de la Información, Máster en Museología, académica e investigadora de la Escuela de Bibliotecología, Documentación e Información de la Universidad Nacional, Costa Rica. Correo electrónico: lucrecia.barboza.jimenez@una.ac.cr

Giannina Ocampo Bermúdez: Licenciada en Bibliotecología con énfasis en Ciencias de la Información, Máster en Ciencias de la Educación con énfasis en Docencia, académica e investigadora de la Escuela de Bibliotecología, Documentación e Información de la Universidad Nacional, Costa Rica. Correo electrónico: giannina.ocampo.bermudez@una.ac.cr 